\title{
Incontinência Urinária em Mulheres Praticantes de Musculação
}

\author{
Andreza Teixeira de Melo$^{1}$; Rosana Porto Cirqueira²
}

\begin{abstract}
Resumo: A International Continence Society, afirma que a Incontinência Urinária é qualquer perda involuntária de urina, classificada em três tipos: Urinária por urgência, urinária mista, Incontinência urinária de esforço. $\mathrm{O}$ objetivo deste estudo foi verificar a presença de incontinência urinaria em mulheres praticantes de musculação. Trata-se de um estudo transversal, realizados em quatro academias credenciadas em vitória da conquista com mulheres acima de 18 anos a amostra foi composta por 30 participantes. Os dados do estudo foram tabulados e processados pelo software Statistical Package for the Social Sciences 22.0 para o Windows. As tabelas foram plotadas no Excel. Teste do qui-quadrado de Pearson (verificar se a presença de incontinência urinaria está correlacionada à prática de atividade física, ao fato das mulheres terem filhos e ao tipo de parto). A presença de filhos foi maior no grupo das incontinentes quando comparadas com as continentes, com $8(88,9 \%)$ e $5(23,8 \%)$ dos casos, respectivamente. $E$ foi verificada correlação estatística significante entre os grupos $(p=0,02)$. quanto ao tipo de parto foi realizado apenas com o total de mulheres que já passaram por gestação 13 (43,3\%). Enquanto nas mulheres diagnosticadas com incontinência urinária $5(62,5 \%)$ realizaram parto vaginal, $3(60,0 \%)$ das continentes realizaram parto cesárea. A diferença para essa distribuição foi verificada estatisticamente $(p=0,04)$. Conclui-se que não houve associação estatisticamente significante entre a prática de musculação e a incontinência urinaria.
\end{abstract}

Palavras-chave: Assoalho pélvico. Incontinência Urinária. Musculação.

\section{Urinary Incontinence in Women Bodybuilding}

\begin{abstract}
The International Continence Society claims that urinary incontinence is any involuntary loss of urine, classified into three types: urinary urgency, mixed urinary incontinence, stress urinary incontinence. The objective of this study was to verify the presence of urinary incontinence in women practicing bodybuilding. It is a crosssectional study, carried out in four accredited academies in victory of conquest with women over 18 years of age. The sample consisted of 30 participants. The study data were tabulated and processed by Statistical Package for Social Sciences 22.0 for Windows. The tables were plotted in Excel. Pearson's chi-square test (check whether the presence of urinary incontinence correlates with physical activity, women having children, and type of delivery). The presence of children was higher in the incontinent group when compared to the continents, with $8(88.9 \%)$ and $5(23.8 \%)$ cases, respectively. A statistically significant correlation was verified between the groups $(p=0.02)$. the type of delivery was performed only with the total number of women who had already undergone pregnancy $13(43.3 \%)$. While in women diagnosed with urinary incontinence $5(62.5 \%)$ performed vaginal delivery, 3 $(60.0 \%)$ of the continents performed cesarean delivery. The difference for this distribution was statistically verified $(\mathrm{p}=0.04)$. It was concluded that there was no statistically significant association between bodybuilding and urinary incontinence.
\end{abstract}

Keywords: Pelvic floor. Urinary incontinence. Bodybuilding.

${ }^{1}$ Docente do curso de Fisioterapia da Faculdade Independente do Nordeste (FAINOR) - Vitória da Conquista (BA), Brasil. Especialista, porto_rosana@yahoo.com.br.

${ }^{2}$ Graduanda do curso de Fisioterapia da Faculdade Independente do Nordeste (FAINOR) - Vitória da Conquista (BA), Brasil, andreza1906melo@gmail.com.

Id on Line Rev. Mult. Psic. V.12, N. 42, , Supl. 1, p. 525-535, 2018
Edição eletrônica em http://idonline.emnuvens.com.br/id 


\section{Introdução}

O assoalho pélvico corresponde ao conjunto das partes moles que fecham a pelve suportando o peso das vísceras em ortostase. É constituído por músculos, fáscias e ligamentos que proporcionam suporte aos órgãos abdominais e pélvicos, contribuindo no controle da continência urinária e fecal, equilibrando os efeitos da pressão intra-abdominal, participando na função sexual e na passagem do feto durante o parto (OLIVEIRA, 2016). A disfunção do assoalho pélvico impede a função adequada dos órgãos podendo gerar enfraquecimento, o que compromete de forma significativa a atividade dessa musculatura, podendo causar diversas disfunções como a incontinência urinária (ALMEIDA et al., 2011).

A International Continence Society (ICS) afirma que a Incontinência Urinária (IU) é qualquer perda involuntária de urina. Sendo classificada em três tipos mais comuns, Incontinência Urinária por Urgência (IUU) na qual as perdas de urina estão associadas a episódios de urgência, ou seja, a uma vontade súbita e intensa de urinar, Incontinência Urinaria Mista (IUM) que está relacionado a combinação de sintomas da incontinência urinária de esforço e de incontinência urinária de urgência, e por fim, a Incontinência Urinária Esforço (IUE) que é definida como perda involuntária de urina aos esforços, tosse ou espirro e afeta de forma negativa a vida das mulheres é a ocorrência mais comum entre as mulheres (NAVES et al., 2016).

A Organização Mundial da Saúde (OMS) traz que a incontinência urinária afeta mais de 200 milhões de pessoas no mundo, sendo destacado como um problema de saúde pública. A prevalência geral da IU pode variar de 5 a $69 \%$ nas mulheres, onde no Brasil, a prevalência é maior para o sexo feminino correspondendo $26,2 \%$ da população e de $11,8 \%$ na população masculina (MARTINES; DAMBROS; TAMANINI, 2014).

Condições bem definidas, como o peso corporal elevado e o peso do útero na gestação, partos, cirurgias pélvicas e o hipoestrogenismo, podem promover ou gerar deficiência na função dos músculos pélvicos nessas mulheres. Recentemente estudos apresentam que fatores que podem aumentar a pressão intra-abdominal, como: tosse crônica, constipação intestinal com esforço crônico para defecar e atividade física extenuante, também podem levar a desencadear condições de incontinência urinária (ALMEIDA et al., 2011).

Embora a realização de exercício físico regular gere inúmeros benefícios, na qualidade de vida das mulheres, estudos vem evidenciando que a execução exagerada e 
inadequada destas atividades podem ter como consequência o surgimento da incontinência urinária, uma disfunção do assoalho pélvico que pode ser adquirida pela prática de musculação, isso pelo uso de cargas excessivas, que geram uma sobrecarga nas musculaturas da área abdominal e pélvica com consequente enfraquecimento, fazendo com que possa surgir outras manifestações uroginecológicas e a ocorrência de patologias (FERREIRA et al., 2015).

A IU é incômoda e vergonhosa, um problema que vem aumentando significavelmente e apesar de esta disfunção estar associada ao envelhecimento fator que vem mudando e se tornando um problema cada vez mais presente entre mulheres jovens, e fisicamente ativas, onde a grande maioria são mulheres. Desta forma apenas uma pequena quantidade procura um serviço médico decorrente da perda de urina (SILVA et al., 2018).

Através da temática abordada, observa-se a importância de identificar as mulheres que praticam musculação regularmente apresentam ou não maior probabilidade de desenvolver disfunções como a incontinência urinária, sendo que essas disfunções geram consequências físicas, psicológicas e sociais (SILVA et al., 2018).

Desta forma o estudo irá contribuir para a conscientização de mulheres e profissionais de academias, sobre a importância de se atentar ao uso correto de equipamentos para que não gere sobrecarga na musculatura pélvica, e que a musculação tenha ação de auxiliar no fortalecimento dessa musculatura, evitando assim danos indesejáveis no futuro.

Considerando o exposto, torna-se relevante avaliar a incontinência urinaria em mulheres de modo a elucidar sua relação com a musculação já que podem estar diretamente afetando a qualidade de vida desta população. Sendo assim, o estudo tem como objetivo analisar a presença de incontinência urinária em mulheres praticantes de musculação.

\section{Metodologia}

Trata-se de um estudo transversal descritivo e analítico, de abordagem quantitativa, Foram incluídas mulheres acima de dezoito anos que praticam musculação regularmente, adotados os seguintes critérios de exclusão: mulheres que já apresentaram alguma patologia que comprometesse a função da musculatura pélvica e que se recusaram a assinar o Termo de Consentimento Livre Esclarecido (TCLE), além de apresentarem idade superior a 50 anos 
Este estudo será realizado em quatro academias credenciadas em Vitória da Conquista, um município brasileiro do Estado da Bahia, cuja população, conforme o IBGE (2013) é de 348 718 habitantes, o que a faz dela a terceira maior cidade do estado, atrás de Salvador e Feira de Santana, e a quarta do interior do Nordeste. Possui uma área de 3.204,257 km² (IBGE, 2013).

O local da pesquisa foi escolhido por conveniência, visto que oferece boas estimativas de características sociodemográficas e socioeconômicas e pela facilidade de acesso aos dados.

A população do estudo foi composta por 30 mulheres praticantes de musculação das quatro academias credenciadas de Vitória da Conquista-BA. A coleta de dados foi realizada no mês de outubro de 2018.

Neste momento foram incluídas mulheres acima de dezoito anos que praticam musculação regularmente, adotados os seguintes critérios de exclusão: mulheres que já apresentou alguma patologia que compromete a função da musculatura pélvica, que se recusaram a assinar o Termo de Consentimento Livre Esclarecido (TCLE) e que apresentaram idade superior a 50 anos, além das que porventura apresentarem respostas contraditórias.

Foram aplicados um instrumento previamente elaborado pelos autores desse estudo sendo o questionário sociodemográfico e também foi utilizado o instrumento de coleta de dados questionário ICIQ-SF International Consultation on Incontinence Questionnaire- Short Form, validado para o português, desenvolvido para avaliar o impacto da IU na qualidade de vida e a qualificação da perda urinária dos pacientes analisados. O ICIQ-SF é composto de quatro questões que avaliam a frequência, a gravidade e o impacto da IU, além de um grupo de oito itens relativos a causas ou situações de IU vivenciadas pelos respondentes o escore é resultado da soma das questões 3, 4 e 5, variando de 0 a 21 pontos, onde 0 ponto demonstra nenhum impacto, 1 a 3 pontos equivale a um impacto leve, 4 a 6 pontos impacto moderado, 7 a 9 pontos impacto grave e 10 ou mais pontos impacto muito grave. Quanto maior o escore maior a severidade da perda urinária e o impacto na qualidade de vida.

Este estudo foi anteriormente aprovado pelo Comitê de Ética e Pesquisa da Faculdade Independente do Nordeste CEP/FAINOR sob o número do parecer: 2.769 .982 obedecendo a Resolução 466/2012 do Conselho Nacional de Saúde em todas suas etapas.

Os dados obtidos neste estudo foram tabulados e processados pelo software Statistical Package for the Social Sciences- SPSS 22.0 para o Windows. As tabelas foram plotadas no Excel. Teste do qui-quadrado de Pearson (pírson) (verificar se a presença de IU está 
correlacionada à prática de atividade física, ao fato das mulheres terem filhos e ao tipo de parto). será adotado o nível de $\mathrm{p}<0,05$ para a significância estatística.

\section{Resultados}

A Caracterização das mulheres que praticam musculação de acordo com as variáveis socioeconômicas e demográficas, seguem apresentada na Tabela 1.

Tabela 1. Características sociodemográficas da amostra. Vitoria da Conquista - BA, 2018.

\begin{tabular}{|c|c|c|}
\hline \multicolumn{3}{|l|}{ Características } \\
\hline Idade, $\operatorname{anos}\left(M^{1} \pm D P^{2}\right)$ & \multicolumn{2}{|c|}{$32,20 \pm 9,15$} \\
\hline \multicolumn{3}{|l|}{ Estado civil, $n \%$} \\
\hline Casada & 13 & 43,3 \\
\hline Solteira & 16 & 53,3 \\
\hline Viúva & 1 & 3,3 \\
\hline \multicolumn{3}{|l|}{ Cor, $n \%$} \\
\hline Parda & 15 & 50,0 \\
\hline Negra & 5 & 16,7 \\
\hline Branca & 10 & 33,3 \\
\hline \multicolumn{3}{|l|}{ Escolaridade, $n \%$} \\
\hline Fundamental incompleto & 6 & 20,0 \\
\hline Ensino Médio Completo & 11 & 36,7 \\
\hline Nível Superior incompleto & 6 & 20,0 \\
\hline Nível Superior Completo & 7 & 23,3 \\
\hline \multicolumn{3}{|l|}{ Frequência da prática, $n \%$} \\
\hline 2 a 3 vezes & 3 & 10,0 \\
\hline 4 a 5 vezes & 19 & 63,3 \\
\hline Acima de 5 vezes & 7 & 23,3 \\
\hline \multicolumn{3}{|l|}{ Tempo que pratica, $n \%$} \\
\hline Entre 1 e 12 meses & 14 & 46,7 \\
\hline Entre 12 e 24 meses & 9 & 30,0 \\
\hline Entre 25 e 36 meses & 2 & 6,7 \\
\hline Acima de 36 meses & 5 & 16,7 \\
\hline \multicolumn{3}{|l|}{ Possui filhos, $n \%$} \\
\hline Não & 17 & 56,7 \\
\hline Sim & 13 & 43,3 \\
\hline \multicolumn{3}{|l|}{ Tipo de parto, $n \%$} \\
\hline Vaginal & 7 & 23,3 \\
\hline Cesária & 6 & 20,0 \\
\hline
\end{tabular}

${ }^{1}$ Média; ${ }^{2}$ Desvio Padrão Amostral; Fonte: Dados da pesquisa 
Entre as participantes da pesquisa $(\mathrm{N}=30)$, a média de idade encontrada foi de $32,20( \pm$ 9,15), com idade máxima de 50 anos e mínima de 18.

Pode-se observar que a maioria das mulheres participantes eram solteiras dessa forma que amostra apresentou estado civil predominantemente casada $16(53,3 \%)$, de cor parda 15 $(50,0 \%)$, com ensino médio completo $11(36,7 \%)$. Quanto a prática de atividade física a frequência predominante foi de 4 a 5 vezes por semana 19 (63,3\%), realizada por um tempo entre1 e 12 meses $14(46,7 \%)$.

Quanto aos antecedentes obstétricos, 17 (56,7\%) não tem filhos e as que possuem 13 $(43,3 \%), 7(23,3 \%)$ realizaram parto vaginal e $6(20,0)$ parto cesárea.

Das 30 mulheres praticantes de atividade física entrevistadas, 30\% (9) delas, foram classificadas como incontinentes pelo resultado do score do ICQ-SF. (Gráfico 1).

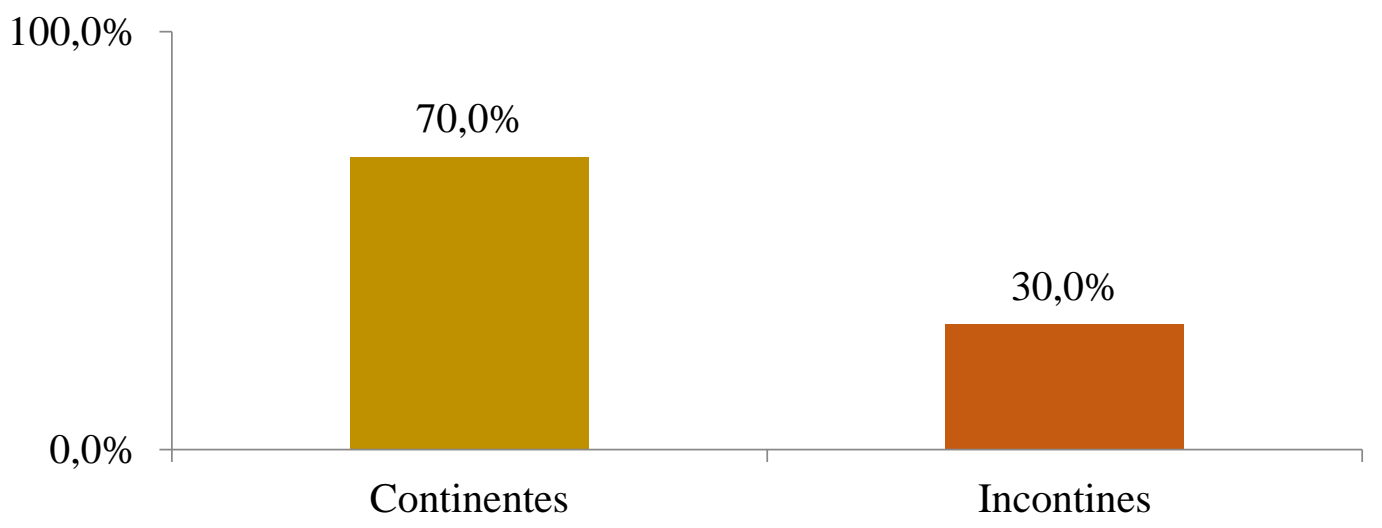

Gráfico 1. Prevalência de Incontinência urinária. Vitória da Conquista - VA, 2018.

Fonte: Dados da pesquisa

Dentre essas que foram classificadas como incontinentes a maioria $5(16,7 \%)$ afirmaram perder urina duas a três vezes por semana e se tratando da quantidade de urina que perdem, $3(10,0 \%)$ afirmaram perda uma vez ao dia e apenas $1(3,3 \%)$ uma vez por semana ou menos.

A quantidade de urina perdida na maioria dos casos foi pequena $6(20,0 \%)$. Quanto ao impacto da incontinência urinária no cotidiano, observou-se que $5(16,7 \%)$ afirmaram ser do tipo "grave "e das 30 entrevistadas, 25 (83,3\%) afirmam não fazer uso de protetor diário, conforme observado na tabela 2. 
Tabela 2. Características das incontinência urinária de mulheres praticantes de atividade física . Vitoria da Conquista - BA, 2018.

\begin{tabular}{lcc}
\hline Características & n & \% \\
\hline Com que frequência perde urina & & \\
$\quad$ Nunca & 21 & 70,0 \\
Uma vez por semana ou menos & 1 & 3,3 \\
Duas ou três vezes por semana & 5 & 16,7 \\
Uma vez ao dia & 3 & 10,0 \\
Quantidade de urina que perde & 6 & 20,0 \\
$\quad$ Pequena & 21 & 70,0 \\
Não perde & 3 & 10,0 \\
Média & & \\
Impacto da IU1 no cotidiano & 21 & 70,0 \\
Nada & 1 & 3,3 \\
Leve & 3 & 10,0 \\
Moderado & 5 & 16,7 \\
Grave & & \\
Uso de protetor diário & 5 & 16,7 \\
Sim & 5 & 83,3 \\
$\quad$ Não
\end{tabular}

${ }^{1}$ Incontinência Urinária; Fonte: Dados da pesquisa.

Com intuito de apresentar os resultados da ocasião em que as mulheres praticantes de atividade de alto impacto mais perdem urina no dia a dia foi esboçado no gráfico 2 .

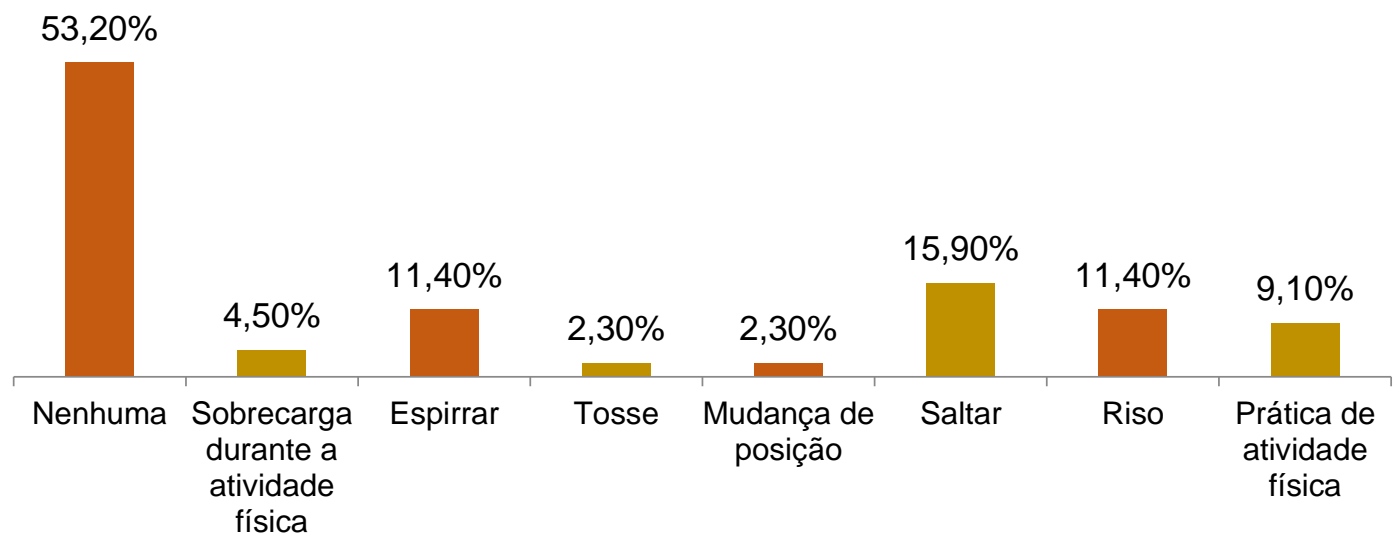

Gráfico 2. Situação em que mulheres praticantes de atividade física mais perdem urina. Vitória da Conquista - BA, 2018

Fonte: Dados da pesquisa 
Infere-se que em $15,90 \%$ dos casos perdem urina ao saltar, enquanto que o Riso e espirro, foram responsáveis por $11,40 \%$ dos casos, seguida ela prática de atividade física, com $9,10 \%$.

Foram analisados quatro possíveis fatores de risco para incidência de perda de urina, dos quais frequência em que realiza a atividade física semanalmente, o tempo em que a prática é realizada, a ocorrência de gestação, bem como o tipo de parto. Nota-se que em ambos os grupos a frequência da prática é de 4 a 5 vezes por semana, sendo $15(71,4 \%)$ para as continentes e $4(50,0 \%)$ para as incontinentes, não sendo encontrado diferença estatística significante entre os grupos $(\mathrm{p}=0,529)$. Assim como na frequência da prática, não foi verificada distribuição tendenciosa para o tempo em que a atividade física é realizada, onde observou-se predominância em 1 a 12 meses nos dois grupos, com 10 (47,6\%) nas continentes e 4 (44,4\%) nas incontinentes, não houve correlação estatisticamente significante $(0,827)$. (Tabela 3$)$.

Tabela 3. Correlação entre a incontinência urinária e dados obstétricos e sobre a atividade física da amostra. Vitória da Conquista - BA. 2018

\begin{tabular}{lccc}
\hline Variáveis, $n(\%)$ & $\begin{array}{c}\text { Continentes } \\
(\mathrm{n}=21)\end{array}$ & $\begin{array}{c}\text { Incontinentes } \\
(\mathrm{n}=9)\end{array}$ & $p^{*}$ \\
\hline $\begin{array}{l}\text { Frequência semanal da } \\
\text { prática de atividade física }\end{array}$ & & & \\
2 a 3 vezes & $2(9,5)$ & $1(12,5)$ & \\
4 a 5 vezes & $15(71,4)$ & $4(50,0)$ & 0,529 \\
$\quad$ Acima de 5 vezes & $4(19,0)$ & $3(37,5)$ & \\
Tempo que pratica a atividade & & & \\
$\quad$ Entre 1 e 12 meses & $10(47,6)$ & $4(44,4)$ & 0,827 \\
Entre 12 e 24 meses & $7(33,3)$ & $2(22,2)$ & \\
Entre 25 e 36 meses & $1(4,8)$ & $1(11,1)$ & \\
$\quad$ Acima de 36 meses & $3(14,3)$ & $2(22,2)$ & \\
Possui filhos & & & \\
$\quad$ Não & $16(76,2)$ & $1(11,1)$ & \\
Sim & $5(23,8)$ & $8(88,9)$ & \\
Tipo de parto & & & \\
Não possui filhos & $16(76,2)$ & $1(11,1)$ & \\
Parto Vaginal & $3(14,3)$ & $4(44,4)$ & \\
Parto Cesária & $2(9,5)$ & $4(44,4)$ & \\
\hline
\end{tabular}

Fonte: Dados da pesquisa. * Teste do qui-quadrado de Pearson.

Em contrapartida, o histórico obstétrico das entrevistadas se mostrou diferente entre os grupos. A presença de filhos foi maior no grupo das incontinentes quando comparadas com 
as continentes, com $8(88,9 \%)$ e $5(23,8 \%)$ dos casos, respectivamente. E foi verificada correlação estatística significante entre os grupos $(p=0,02)$.

O cruzamento de dados quanto ao tipo de parto foi realizado apenas com o total de mulheres que já passaram por gestação 13 (43,3\%). Enquanto nas mulheres diagnosticadas com incontinência urinária 5 (62,5\%) realizaram parto vaginal, $3(60,0 \%)$ das continentes realizaram parto cesárea. A diferença para essa distribuição foi verificada estatisticamente $(p=0,04)$.

\section{Discursão}

Os resultados obtidos no presente estudo demostram que não houve associação, significância estatística entre incontinência urinaria em mulheres que praticam musculação, no entanto estudos realizados no país demostram que existe associação entre as variáveis dessa forma sugere-se que esse estudo não tenha tido associação devido a omissão de informação dessas mulheres pelo fato de se sentir constrangidas.

O estudo teve significância em outras variáveis que não teve relação com a musculação sendo assim não gerou resultados partindo do objetivo principal não respondendo ao mesmo.

Com relação aos músculos do assolho pélvico existe pouco conhecimento acerca do funcionamento dessa musculatura durante a prática desportiva. Na maioria dos exercícios em que ocorre aumento da pressão intra-abdominal, não existe contração voluntária desses músculos, motivo que explicaria a perda involuntária de urina (ARAUJO et al.,2015).

Determinados autores consideram que algumas atividades esportivas de alto impacto fazem com que ocorra a sobrecarga, e tendem a levar ao enfraquecimento da musculatura perineal, de modo que gere aumento no risco de aparecimento de episódios de incontinência urinaria (BO, 2004; PÓSWIATA; SOCHA; OPARA, 2014).

Contradizendo aos resultados obtidos no presente estudo anteriormente, Patrizzi et al. Analisou 3 grupos que praticavam atividade física, entre outras verificou a musculação. $O$ grupo que obteve maior pera urinária foi a musculação $(61,1 \%)$, seguindo dos exercícios aeróbios $(41,6 \%)$ e da natação $(25 \%)$.

Segundo o estudo de Rincon (2015) a IU foi significativamente associada à eventos obstétricos como quantidade e tipo de parto, episiotomias, peso do bebe ao nascer são os principais fatores de risco para a IU. Assim, o trauma perineal ocorrido por ocasião do parto é 
o grande responsável pelo aparecimento desta disfunção. $\mathrm{O}$ estudo traz que presença de filhos foi maior no grupo das incontinentes quando comparadas com as continentes, com $8(88,9 \%)$ e $5(23,8 \%)$ dos casos, respectivamente, analisando as mulheres que apresentam incontinência urinária $5(62,5 \%)$ realizaram parto vaginal, $3(60,0 \%)$ das continentes realizaram parto cesárea.

A literatura traz que mulheres submetidas a parto vaginal apresentam maior prevalência de IU pós-parto quando comparadas a mulheres submetidas à cesárea (LEROY et al.,2016).

Em relação ao impacto da incontinência urinária no cotidiano, observou-se que $5(16,7 \%)$ afirmam ser do tipo "grave” relataram que a IU afeta sua vida. Rodrigues et al., (2016) em seu estudo aponta que a IU tem um impacto negativo importante na vida das participantes.

Apesar de que o presente estudo apresenta limitações no que se refere ao tamanho da amostra, e também ao local adequado foi possível notar que características importantes relacionadas a antecedentes obstétricos e presença de filhos com a incontinência urinaria

\section{Conclusão}

Conclui-se que não houve associação estatisticamente significante entre a prática de musculação e a incontinência urinaria, no entanto, e necessário que seja realizado estudos que possa ser melhor controlado essa questão da omissão das respostas isso poderia ser amenizado com um local adequado para responder os questionários.

Outros estudos se fazem necessários para investigar os fatores de risco o que viabilizará a elaboração e implementação de ações em prevenção e promoção de saúde, levando informações aos praticantes e profissionais, afim de minimizar os agravos gerados pela deficiência da musculatura do assoalho pélvico evitando assim danos indesejáveis no futuro. Conscientizando os próprios profissionais a ter um olhar mais atencioso para o condicionamento e treinamento dessas mulheres, visando reduzir a prevalência dessa condição.

\section{Referências}

ALMEIDA, M. B. A, et al. Disfunções de assoalho pélvico em atletas. Revista Femina, Belo Horizonte, v. 39, n. 8, p.395-402, ago. 2011. 
ARAUJO, M. Poli, et al. Evaluation Of Athletes'pelvic Floor: Is There A Relation With Urinary Incontinence? Revista Brasileira de Medicina do Esporte, v. 21, n. 6, p. 442-446, 2015.

FERREIRA, T. C. R, et al. Avaliação da força muscular do assoalho pélvico em mulheres sedentárias e que praticam atividade física. Revista da Universidade Vale do Rio Verde, v. 13, n. 2, p. 450-464, 2015.

IBGE. Senso 2013. Disponível em:<https://censo2010.ibge.gov.br/>.

LEROY, Lígia da Silva; LÚCIO, Adélia; LOPES, Maria Helena Baena de Moraes. Risk factors for postpartum urinary incontinence. Revista da Escola de Enfermagem da USP, v. 50, n. 2, p. 200-207, 2016.

MARTINES, G. A.; DAMBROS, M.; TAMANINI, J.T.N.Effect of strength training on the gain of muscle strength in the lower limbs of women with stress urinary incontinence. Revista Pan-Amazônica de Saúde, v. 5, n. 4, p. 29-36, 2014.

NAVES, P. P.et al. Avaliação Da Qualidade De Vida Em Mulheres Com Sintomas De Incontinência Urinária De Esforço Praticantes De Atividade Física. Revista Inspirar Movimento \& Saude, v. 8, n. 1, 2016.

OLIVEIRA, H.G.A. Efeitos do método Pilates nas disfunções do assoalho pélvico: Uma revisão sistemática. 2016. 22 f. TCC (Graduação) - Curso de Fisioterapia, Universidade Estadual da Paraĺba, Campina Grande, 2016.

PÓSWIATA, A.; SOCHA, T.; OPARA, J. Prevalence of Stress Urinary Incontinence in Elite Female Endurance Athletes. Journal of Human Kinetics, v. 44, p. 91-96, 2014.

SILVA, Leidiany Bueno da et al. Disfunções urinárias em mulheres praticantes de atividade física em academias-um estudo transversal. Revista Pesquisa em Fisioterapia, v. 8, n. 1, p. 71-78, 2018.

\section{Como citar este artigo (Formato ABNT):}

MELO, Andreza Teixeira de; CIRQUEIRA, Rosana Porto. Incontinência Urinária em Mulheres Praticantes de Musculação. Id on Line Rev.Mult. Psic., 2018, vol.12, n.42, Supl. 1, p. 525-535. ISSN: 1981-1179.

Recebido: 08/11/2018;

Aceito: $12 / 11 / 2018$ 